\title{
HUFFMAN CODING AND CHAINS IN PARTITION LATTICES
}

\author{
STEPHAN FOLDES
}

\begin{abstract}
The Huffman coding algorithm is interpreted in the context of the lattice of partitions of the source alphabet. Maximal chains in the partition lattice correspond to linear extensions of tree orders, and those among the chains that exhibit a simple greedy property correspond precisely to executions of the Huffman algorithm.
\end{abstract}

The input to the Huffman algorithm of information theory is a non-empty finite set $S$, called source alphabet, together with a map $p$ associating to each source symbol $i \in S$ a non-negative real number $p(i)$. For each subset $A \subseteq S$, we also write $p(A)$ for

$$
\sum_{i \in A} p(i)
$$

In this paper, we deal only with the Huffman coding algorithm for binary coding, as the corresponding chains in the partition lattice will not in general be maximal chains otherwise.

The Huffman algorithm constructs a binary tree whose terminal nodes are the source symbols, source symbol $i$ being at distance $l_{i}$ in the tree, such that the sum

$$
\sum_{i \in S} p(i) l_{i}
$$

is minimal among all binary trees with terminal node set $S$. (A binary Huffman code is then produced by topologizing the tree, designating the sons of each nonterminal node as left or right son, and recording the left-right direction sequences of the path to each terminal node from the root as a $0-1$ sequence.) The Huffman algorithm is non-deterministic, in the sense that arbitrary choices made during its execution may produce different, even non-isomorphic, but equally optimal trees. The following easy observation is made as the first argument in usually given proofs of optimality:

Observation. There is a binary tree with terminal node set $S$ such that there are two distinct $j, k \in S$ with

$$
l_{j}=l_{k}=\max \left\{l_{i}: i \in S\right\}
$$

and

$$
\max (p(j), p(k)) \leq \min \{p(i): i \in S, i \neq j, i \neq k\} .
$$

$M S C$ (2010): primary 68P30, 06C10.

Keywords: Huffman algorithm, partition lattice, maximal chain, binary tree, linear extension, CD basis. 
It is useful to consider also the sum (1) not only for binary trees, but also for binary forests with terminal node set $S$ (where $l_{i}$ is the distance of $i$ from the root of the tree component to which $i$ belongs). Note that we omit the usual assumption that the sum of the various $p(i)$ is 1 , as this plays a role only in the interpretation of the input and the output of the Huffman algorithm, but not in the procedure itself. The Huffman algorithm constructs the optimal binary tree by building a sequence of binary forests with the same terminal node set $S$, starting with the forest in which every tree component has only one node (a source symbol), moving at each step from a given forest to a new one with one less tree component, by including two of the tree components $T_{1}, T_{2}$ in a single new tree component $T$ so that the root of $T$ (the only new node in the next forest) will have the roots of $T_{1}$ and $T_{2}$ as its sons. Cleraly, the end result is a tree, and the number of forests involved in the sequence is the number of source symbols. The specificity of the Hufmann algorithm, which turns out to guarantee the optimality of the tree constructed, is that at each step the tree components $T_{1}$ and $T_{2}$ are chosen so that the sum of the numbers $p(i)$ taken over the terminal nodes of the two chosen tree components is minimal, among all possible choices of two tree components.

It is irrelevant what the nodes of the forests actually are; therefore, to have a canonical representation of the forests, we shall suppose that each terminal node is a singleton containing a source symbol, and each node $i s$ simply the set of those source symbols at the terminal nodes that are its descendants (which set does not change from the stage when the node is added until the procedure terminates). Then, the node set of the tree resulting at the end of an execution of the Huffman algorithm is nothing else, in the terminology of comparable-or-disjoint set families (see [1-3]), than a CD basis minus the empty set, in the lattice of subsets of the source alphabet. In fact, at each stage of the algorithm what we have is a CDindependent set $F$ of non-empty sets of source symbols, such that for each maximal member $X$ of $F$, the set

$$
\{Y \in F: Y \subseteq X\}
$$

together with $\emptyset$ is a CD basis of the lattice of subsets of $X$. This is in fact a complete characterization of the Huffman algorithm. The algorithm can also be described as follows:

Huffman Algorithm. Given a non-empty finite set $S$ of $n$ elements, with a non-negative real number $p(i)$ assigned to each $i \in S$. Let $\mathcal{C}$ be the set of maximal chains in the lattice of partitions of a finite non-empty set $S$, construct a sequence $F_{0} \subset F_{1} \subset F_{2} \subset \ldots$ of families of subsets of $S$, such that

(i) $F_{0}$ is the family of all singletons,

(ii) while $S \notin F_{k}$ let $F_{k+1}$

consist of the members of $F_{k}$ and of the union of two distinct maximal members $A \cup B$ of $F_{k}$ such that the sum

$$
\sum_{i \in A \cup B} p(i) l_{i}
$$

is of maximal value over all choices of distinct maximal members $A, B$ of $F_{k}$.

Then, the sequence stops at stage $n-1, F_{0} \subset F_{1} \subset \cdots \subset F_{n-1}$ where $F_{n-1} \cup\{\emptyset\}$ is a $C D$ basis in the lattice of subsets of $S$, minimizing over all $C D$ bases $F$ the 
sum

$$
\sum_{i \in S} p(i)[\operatorname{Card}\{A \in F: A \neq S, i \in A\}]=\sum_{\substack{A \in F \\ A \neq S}} p(A) .
$$

Given any finite non-empty set $S$, say with $n$ elements, consider a CD basis $F$ of the lattice of subsets of $S$. Then, the non-empty members of $F$ constitute a binary tree $T$ with root $S$, the terminal nodes of which are the singleton subsets of $S$. Consider the set $F^{\prime}$ of non-singleton members of $F$, and any linear extension $\lambda$ of the subset relation (partial order) of $F^{\prime}$. Cleraly, $F^{\prime}$ has $n-1$ members, let these be enumerated $A_{1}, \ldots, A_{n-1}$ in the increasing order of $\lambda$. Then, to $\lambda$, we can associate a maximal chain $\Pi_{0}<\Pi_{1}<\cdots<\Pi_{n-1}$ in the lattice of partitions of $S$, where for $1 \leq k \leq n-1$, the partition $\Pi_{k}$ is obtained from $\Pi_{k-1}$ by replacing with $A_{k}$ those two (uniquely determined) partition classes of $\Pi_{k-1}$ the union of which is $A_{k}$. In fact, all maximal chains in the partition lattice can be obtained this way, which we can also state in terms of the reverse construction, in Proposition 1 below. Let $\mathcal{C}$ denote the set of maximal chains in the lattice of partitions of a finite non-empty set $S$. For each maximal chain $K$ of partitions $\Pi_{0}<\Pi_{1}<\ldots$ of $S$ and each non-singleton set $A$ appearing as a class in any member of $K$, call the positive integer $\min \left\{k: 0<k, A\right.$ is a class of $\left.\Pi_{k}\right\}$ the index of first appearance of $A$ in $K$. Note that no two different sets can have the same index of first appearance, and thus the non-singleton classes of the various members of $K$ are linearly ordered according to their index of first appearance.

Proposition 1. Let $\mathcal{C}$ be the set of maximal chains in the lattice of partitions of a finite non-empty set $S$. For each $C D$ basis $F$ of the lattice of subsets of $S$, let $\Lambda_{F}$ be the set of linear extensions of the set containment order on the set $F^{\prime}$ of non-empty, non-singleton members of $F$, and let $\Lambda$ be the union of all the $\Lambda_{F}$ for the various $C D$ bases $F$.

Then, the map, which to each maximal chain $K$ of partitions $\Pi_{0}<\Pi_{1}<\ldots$ of $S$ associates the set of non-singleton classes of the various members of $K$, linearly ordered according to their indices of first appearance in $K$, is a bijection from $\mathcal{C}$ to $\Lambda$.

Greedy Chain Characterization. The sequence $F_{0} \subset F_{1} \subset \cdots \subset F_{n-1}$ of sets of source symbols constructed by any execution of the Huffman Algorithm on an n-element finite, non-empty set $S$, with a non-negative real number $p(i)$ assigned to each $i \in S$, defines a maximal chain $\Pi_{0}<\Pi_{1}<\cdots<\Pi_{n-1}$ in the lattice of partitions of $S$, where for $1 \leq k \leq n-1$, the partition $\Pi_{k}$ is obtained from $\Pi_{k-1}$ by replacing with the only set $C \in F_{k} \backslash F_{k-1}$ the two classes $A, B$ of $\Pi_{k-1}$ for which $C=A \cup B$.

A maximal chain $\Pi_{0}<\Pi_{1}<\cdots<\Pi_{n-1}$ in the lattice of partitions of a finite non-empty set $S$ corresponds to an execution of the Huffman Algorithm if and only if for each $1 \leq k \leq n-1$ the (uniquely determined) class $C \in \Pi_{k} \backslash \Pi_{k-1}$ satisfies $p(C) \leq p(K)$, for the sets $K \in \Pi \backslash \Pi_{k-1}$ for all partitions $\Pi$ covering $\Pi_{k-1}$ in the partition lattice.

Acknowledgement. This paper reports on research carried out while the author, then affiliated with the Tampere University of Technology (Finland), was 
visiting at the University of Miskolc (Hungary). The research was co-funded by Marie Curie Actions and supported by the National Development Agency (NDA) of Hungary and the Hungarian Scientific Research Fund (OTKA, contract number 84593), within a project hosted by the University of Miskolc, Department of Analysis.

The author wishes to thank Sándor Radeleczki for useful comments and discussions.

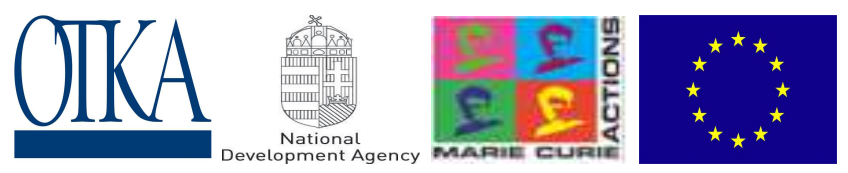

\section{REFERENCES}

[1] G. Czédli and E. T. Schmidt, CDW-independent subsets in distributive lattices, Acta Scientiarum Mathematicarum 75 (2009), 49-53.

[2] G. Czédli, M. Hartmann and E. T. Schmidt, CD-independent subsets in distributive lattices, Publicationes Mathematicae 74 (2009), 127-134.

[3] E. K. Horváth and S. Radeleczki, Notes on CD-independent subsets, Acta Scientiarum Mathematicarum 78 (2012), 3-24.

Stephan Foldes, University of Miskolc, Hungary

e-mail: foldes.istvan@uni-miskolc.hu 